\title{
CONTAMINACIÓN DE LOS PARQUES PÚBLICOS DE LOS DISTRITOS DE LIMA OESTE CON HUEVOS DE Toxocara sp.
}

\author{
Francisco López T. ${ }^{1}$, Amanda Chávez V. ${ }^{2,3}$ y Eva Casas A. ${ }^{2}$
}

\section{Abstract}

The objective of this study was to determine the level of contamination of public parks with Toxocara sp. eggs in Western Lima. Soil and grass samples were collected using the Double W method from April till August 1999 in 123 public parks of the districts of Breña, Jesus María, La Victoria, Lima, Lince, Magdalena del Mar, Miraflores, Pueblo Libre, San Borja, San Isidro, San Luis, San Miguel and Surquillo. The temperature varied between 24.4 and $16.2^{\circ} \mathrm{C}$ and the mean monthly relative humidity was $91.5 \%$. The results showed that 78 parks were positive to Toxocara sp. eggs, which represents a prevalence of $63 \pm 9 \%$. Parks were classified according to the degree of conservation and to the socioeconomic status of the inhabitants. The well, average, and poorly maintained parks had 71,50 , and $50 \%$ of contamination, respectively. Parks located in areas of high socioeconomic status were more contaminated than those located in areas of low socioeconomic status $(69.2,66.6,50.0,50.0$, and $33.3 \%$ in high, medium high, medium, medium low, and low, respectively). Toxocara sp. eggs found in these parks were viable as indicated through lesions develoved by experimentally infected quails. It is necessary to regulate the entrance of dogs in public parks and to inforce the implementation of latrines for pets.

Key words: Toxocara, toxocariosis, zoonosis, public parks

\section{Resumen}

El objetivo del presente estudio fue determinar el nivel de contaminación con huevos de Toxocara sp. de los parques públicos de la zona de Lima Oeste. Muestras de tierra y césped de 123 parques públicos de los distritos de Breña, Jesús María, La Victoria, Lima, Lince, Magdalena del Mar, Miraflores, Pueblo Libre, San Borja, San Isidro, San Luis, San Miguel y Surquillo fueron colectados empleando el método de la Doble W entre los meses de abril y agosto de 1999. La temperatura ambiental varió entre 24.4 a $16.2 \mathrm{C}^{\circ}$ y la humedad relativa media mensual fue de $91.5 \%$. Se encontró 78 parques positivos a huevos de Toxocara sp., resultando una prevalencia de $63 \pm 9 \%$. Se clasificaron los parques de acuerdo al grado de conservación y estrato socioeconómico de sus pobladores. Los parques con buen, mediano y mal estado de conservación presentaron el 71, 50 y $50 \%$ de contaminación, respectivamente. Los parques localizados en zonas de mejor

\footnotetext{
${ }^{1}$ Práctica privada

${ }^{2}$ Laboratorio de Microbiología y Parasitología Veterinaria, FMV-UNMSM

${ }^{3}$ E-mail: a_chavez_g@hotmail.com
} 
nivel socioeconómico se encontraron contaminados en mayor proporción que aquellos localizados en zonas de menor nivel $(69.2,66.6,50.0,50.0$ y $33.3 \%$ para los niveles alto, medio alto, medio, medio bajo y bajo, respectivamente). Se determinó que los huevos de Toxocara sp. se encontraban viables pues produjeron lesiones en codornices infectadas artificialmente. Se hace necesario reglamentar la circulación de perros en parques públicos y la implementación de letrinas para mascotas para evitar que las mascotas defequen libremente en los parques.

Palabras clave: Toxocara, toxocariosis, zoonosis, parque público

\section{INTRODUCCIÓN}

La convivencia del hombre con los animales de compañía predispone a la ocurrencia de una serie de enfermedades zoonóticas (Soulsby, 1987). Dentro de éstas, se encuentran las zoonosis parasitarias como la Toxocara canis y la Toxocara cati, parásitos cosmopolitas que pueden causar problemas de toxocariasis en el humano, especialmente en infantes (Acha y Szyfres, 1989).

El ciclo vital del Toxocara canis es complejo, existiendo cuatro formas de transmisión en los perros: prenatal, calostral, directa y por hospederos paraténicos. A diferencia del $T$. canis, la contaminación con $T$. cati no implica infección prenatal pero si lactogénica y por hospederos paraténicos importantes. Las formas de LMV (larva migrante visceral) y LMO (larva migrante ocular) se presentan en el humano y se deben principalmente a la ingestión de huevos larvados con el segundo estadio de Toxocara sp. (Atías, 1994; Leguía, 1996) que se encuentran diseminados en la tierra y césped de los parques públicos (Georgi y Georgi, 1994).

Se dispone de información sobre grados de contaminación de Toxocara sp. en parques públicos de ciudades importantes. Así, se reporta que el $25 \%$ de parques se encontraba contaminado en la ciudad de Birminngham y el 27\% en Londres (Borg y Woodruff, 1973), el 16\% en Illinois (Paul et $a l .$, 1988), el 17\% en Basrah (Mahdi y Ali,
1993), el 15\% en Jordania (Abo-Shehada y Herber, 1984), el 63\% en Tokushima (Shimizu, 1993) y el $42 \%$ en La Habana (Dumenigo y Gálvez, 1995). En el Perú, los primeros estudios realizados por Guerrero (1975) en parques públicos de Lima Metropolitana dieron un resultado de $24 \%$. Estudios posteriores señalaron niveles de contaminación del 37\% de los parques de la Provincia Constitucional del Callao (Velarde, 1999), 30\% de los parques públicos de los distritos del Cono Sur, $41 \%$ de los parques del Cono Este de Lima (Serrano et al., 2000) y $30 \%$ de los parques del Cono Norte (La Rosa et al., 2001).

La alta prevalencia de Toxocara sp. en perros y gatos, el gran número de huevos que éstos eliminan y su gran resistencia al medio ambiente, principalmente en suelos húmedos, favorecen su supervivencia y contribuyen a la contaminación del suelo, el cual constituiría la principal fuente de infección para el hombre (Acha y Szyfres, 1989). En el Perú, Morales, en 1983 (citado por Reyes et al., 1999), determinó que el $70 \%$ de perros de la zona de Lima Metropolitana estaban infectados por Toxocara sp.

El presente estudio tuvo por objetivo hacer la evaluación del grado de infección de los parques públicos de Lima Oeste como parte final del proyecto de evaluación parasitaria de las grandes áreas de Lima Metropolitana llevada a cabo por el Laboratorio de Parasitología de la Facultad de Medicina Ve terinaria de la Universidad Nacional Mayor de San Marcos. 


\section{Materiales y Métodos}

El presente trabajo evaluó la contaminación de los parques públicos de la zona Oeste de Lima Metropolitana (Breña, Jesús María, La Victoria, Lima, Lince, Magdalena del Mar, Miraflores, Pueblo Libre, San Borja, San Isidro, San Luis, San Miguel y Surquillo).

La recolección de muestras se realizó en el periodo de abril-agosto de 1999 , donde se registró una temperatura mínima de $16^{\circ} \mathrm{C}$, una temperatura máxima de $20.4{ }^{\circ} \mathrm{C}$ y una humedad relativa media de 87 a $94 \%$ (SENAMHI, 2002). El método de recolección de muestras y el análisis de las mismas se hizo siguiendo el protocolo de trabajos previos (Velarde, 1999; Serrano et al., 2000; Cajas, 1999; La Rosa et al., 2001).

El tamaño muestral (123 parques) se determinó usando la fórmula para proporciones en poblaciones finitas (Daniel, 1996). Este número se estratificó teniendo en cuenta el número de parques por cada distrito, realizándose un muestreo al azar simple.

Para determinar la viabilidad de los huevos obtenidos de los parques positivos, estos se incubaron en una solución de bicromato de potasio al $2.5 \%$ por 30 días, con el fin de obtener huevos larvados. Posteriormente, 1,500 de estos huevos fueron inoculados vía oral en cuatro codornices, mientras que otras cuatro se mantuvieron como grupo control. Las codornices se sacrificaron a las 24 horas, 5, 7 y 10 días post infección, con el fin de hallar posibles lesiones hepáticas, pulmonares, etc. La presencia de larvas en las vísceras de las codornices se determinó por el método de Baermann.

\section{Resultados y Discusión}

Los resultados mostraron que el $63 \pm 9 \%$ de los parques públicos de la zona de Lima Oeste se encontraban contaminados con hue- vos de Toxocara sp. El distrito más contaminado fue Breña, donde los cuatro parques muestreados resultaron positivos, en tanto que el distrito menos contaminado fue Surquillo con el $25 \%$ de sus parques contaminados (Cuadro 1).

En trabajos previos llevados a cabo en Lima Metropolitana utilizando un protocolo de trabajo similar, se encontraron prevalencias de $29 \%$ de parques públicos contaminados en el cono Sur de Lima (Cajas, 1999), $41 \%$ en el Cono Este (Serrano et al., 2000), 34\% en el Cono Norte (La Rosa et $a l ., 2001)$ y $37 \%$ en los parques de la Provincia Constitucional del Callao (Velarde, 1999). En el presente trabajo, el $63 \%$ de contaminación fue el más alto debido posiblemente a que en el Cono Oeste de Lima se encuentran muchos parques ubicados en zonas de estratos socioeconomicos más altos, en comparación con otras áreas de Lima. Esto se puede traducir en una mejor conservación de sus parques, con tierras húmedas, umbrosas, coloidales y compactas, que hacen un habitat adecuado para la viabilidad de los huevos del Toxocara sp., en contraste con parques más descuidados con terrenos secos, blandos y arenosos.

En el Cuadro 2 se muestra el porcentaje de parques contaminados, según su estado de conservación. Se nota que los parques en mejor estado de conservación eran los más contaminados ( $71 \%$ de prevalencia). La estructura y composición del suelo juegan un papel muy importante, sobre todo en aquellos parques bien conservados, cuya vegetación mantiene condiciones suficientes de humedad y microclimas favorables para el desarrollo de los huevos de Toxocara sp. Años atrás, Buitrón (1976) encontró una prevalencia elevada de contaminación en áreas con gran número de jardines, áreas de cultivo o huertos, pero en aquellos sectores sin jardines o aquellos terrenos áridos no se obtuvo ninguna muestra positiva. En México, Vásquez et al. (1996) demostraron que los jardines caseros estaban más contaminados (16.9\%) que los parques públicos (10.9\%). 
Cuadro 1. Porcentaje de parques contaminados con huevos de Toxocara sp. del Cono Oeste de Lima Metropolitana. 1999

\begin{tabular}{lccc}
\hline \multirow{2}{*}{ Distritos } & \multicolumn{2}{c}{ No de parques $^{\circ}$} & $\begin{array}{c}\text { Frecuencia } \pm \text { IC }^{1} \\
(\%)\end{array}$ \\
\cline { 2 - 3 } & Muestreados & Positivos & 100 \\
Breña & 4 & 4 & 50 \\
Jesús María & 4 & 2 & 58 \\
La Victoria & 12 & 7 & 50 \\
Lima & 20 & 10 & 50 \\
Lince & 2 & 1 & 50 \\
Magdalena del Mar & 2 & 1 & 62 \\
Miraflores & 13 & 8 & 73 \\
Pueblo Libre & 11 & 8 & 82 \\
San Borja & 11 & 9 & 82 \\
San Isidro & 11 & 9 & 80 \\
San Luis & 5 & 4 & 65 \\
San Miguel & 20 & 13 & 25 \\
Surquillo & 8 & 2 & $63 \pm 9$ \\
\hline & 123 & 78 & \\
\hline
\end{tabular}

${ }^{1}$ Intervalo de confianza del $95 \%$

Cuadro 2. Frecuencia de parques contaminados con huevos de Toxocara sp. en el Cono Oeste de Lima Metropolitana, según sus estados de conservación. 1999

\begin{tabular}{lccc}
\hline \multirow{2}{*}{ Estado del parque } & \multicolumn{2}{c}{$\mathrm{N}^{\mathrm{o}}$ de Parques } & \multirow{2}{*}{$\%$} \\
\cline { 2 - 3 } & Muestreados & Positivos & \\
\hline Bien conservados & 77 & 55 & 71.0 \\
Medianamente conservados & 26 & 13 & 50.0 \\
Mal conservados & 20 & 10 & 50.0 \\
\hline
\end{tabular}

La evaluación de parques clasificados según su ubicación en los cinco estratos socioeconómicos (INEI, 1998) demuestran que los parques agrupados en los estratos socioeconómicos altos y medio altos se encuentran altamente contaminados, mientras que los parques menos contaminados se encuentran en zonas del estrato bajo (Cuadro 3). 
Cuadro 3. Distribución de parques contaminados con huevos de Toxocara sp. del Cono Oeste de Lima Metropolitana, según el estrato socio económico. 1999

\begin{tabular}{|c|c|c|c|c|}
\hline \multirow{2}{*}{ Estrato $^{1}$} & \multirow{2}{*}{ Distrito } & \multirow{2}{*}{$\begin{array}{l}\mathrm{N}^{\circ} \text { de parques } \\
\text { muestreados }\end{array}$} & \multicolumn{2}{|c|}{ Parques positivos } \\
\hline & & & $\mathrm{n}$ & $\%$ \\
\hline Alto & $\begin{array}{l}\text { Lima, Magdalena del Mar, } \\
\text { Miraflores, Pueblo Libre, } \\
\text { San Borja, San Isidro, San } \\
\text { Miguel, Surquillo, Breña }\end{array}$ & 39 & 27 & 69.2 \\
\hline Medio Alto & $\begin{array}{l}\text { Jesús María, La Victoria, } \\
\text { Lima, Lince, Pueblo Libre, } \\
\text { San Luis, San Miguel, } \\
\text { Surquillo }\end{array}$ & 60 & 40 & 66.6 \\
\hline Medio & $\begin{array}{l}\text { Lima, La Victoria, } \\
\text { Magdalena del Mar, San } \\
\text { Miguel, Surquillo }\end{array}$ & 14 & 7 & 50.0 \\
\hline Medio Bajo & $\begin{array}{l}\text { Lima, La Victoria, San } \\
\text { Miguel, Surquillo }\end{array}$ & 7 & 3 & 50.0 \\
\hline Bajo & $\begin{array}{l}\text { La Victoria, San Miguel, } \\
\text { Surquillo }\end{array}$ & 3 & 1 & 33.3 \\
\hline
\end{tabular}

${ }^{1}$ INEI (1998)

Cajas (1999) demuestra que en zonas de estratos socioeconómicos bajos predominan parques en mal estado de conservación. Estos parques debido a la sequedad y composición arenosa de sus suelos, no evidencian las condiciones adecuadas para el desarrollo de los huevos de Toxocara sp.

Estos resultados explican la relación directa que existe entre el estrato socioeconómico y el grado de conservación de los parques (Shimizu, 1993).

Es muy común que la infección en humanos se deba principalmente a la ingestión de los huevos larvados con el segundo estadio de Toxocara sp. (Atías, 1994). Las codornices inoculadas con los huevos larvados dieron resultados positivos, demostrándose la viabilidad de los huevos de Toxocara sp. encontrados en los parques públicos del cono Oeste de Lima Metropolitana.
Se concluye que los distritos de la jurisdicción de Lima Oeste presentan una alta contaminación de huevos de Toxocara sp. $(63 \pm 9 \%)$. Se recomienda que las autoridades distritales realicen charlas informativas, desparasitaciones periódicas de las mascotas, campañas de educación sanitaria, así como facilitar el uso de bolsas plásticas para a la recolección de las deyecciones de los perros en los parques públicos.

\section{Literatura Citada}

1. Abo-Shehada, M.N.; I.V. Herber. 1984. The migration of larval Toxocara canis in mice. II. Post intestinal migration in primary infections. Vet. Parasitol. 17: 75- 83.

2. Acha, P.N.; B. Szyfres. 1989. Zoonosis y enfermedades transmisibles comunes 
al hombre y a los animales. $2^{\mathrm{a} e d . ~} \mathrm{p} 844-$ 850. OPS. Washington D.C.

3. Atías, A. 1994. Parasitología clínica. $3^{\mathrm{a}}$ ed. p 314-318. Ed. Mediterráneo. Chile.

4. Borg, O.; A. Woodruff. 1973. Prevalence of infective Toxocara species in public places. Br. Med. J. 4: 470-472.

5. Buitrón, L.A. 1976. Estudio de la contaminación de áreas de uso público con huevos de Toxocara spp. en el área urbana de Paramonga, distrito de Pativilca, provincia de Chancay, departamento de Lima. Tesis de Bachillerato. Facultad de Medicina Veterinaria, Univ. Nacional Mayor San Marcos. Lima. 25 p.

6. Cajas, U. 1999. Estudio de la contaminación de parques públicos con huevos de Toxocara spp. en los distritos del Cono Sur. Tesis de Médico Veterinario. Facultad de Medicina Veterinaria, Univ. Nacional Mayor de San Marcos. Lima. 77 p.

7. Daniel, W. 1996. Biostadística. Base para el análisis de las ciencias de la salud. $3^{a}$ ed. p 206. Ed. LIMUSA. México.

8. Dumenigo, B.; D. Gálvez. 1995. Soil contamination in Habana city with Toxocara canis eggs. Rev. Cub. Med. Trop. 47: 178-180.

9. Georgi, J.R.; M.E. Georgi. 1994. Parasitología en clínica canina. $4^{\mathrm{a}}$ ed $\mathrm{p}$. 171-178. Ed. Interamericana. México.

10. Guerrero, M.O. 1975. Estudio de la contaminación de parques públicos de Lima Metropolitana con huevos de Toxocara spp. Tesis de Bachiller. Facultad de Medicina Veterinaria, Univ. Nacional Mayor de San Marcos. Lima. 12 p.

11. INEI, 1998. Planos estratificados de Lima Metropolitana a nivel de manzanas según estratos socioeconómicos de hogares. Bol. Estadísticas Geográficas. 9700.002 (1): 10-45.

12. La Rosa, V.; A. Chávez; E. Casas. 2001. Contaminación de parques públicos del Cono Norte con huevos de Toxocara spp. Rev. Inv. Vet. Perú 12: 116-121.
13. Leguía, P. 1996. Enfermedades parasitarias de perros y gatos. p 10-19. Ed De Mar. Lima.

14. Mahdi, N.K.; H.A. Ali. 1993. Toxocara eggs in the soil of public places and schools in Basrah, Iraq. Ann. Trop. Med. Parasitol. 87: 201-205.

15. Paul, A.J.; K.S. Todd; J.A. Di Prietro. 1988. Enviromental contamination by eggs of Toxocara species. Vet. Parasitol. 26: 339-342.

16. Reyes, M.; G. Díaz; J. Elías; K. Rodas; J. Roman; R. Ríos; R. Espino. 1999. Relación entre toxocariasis canina domiciliaria y larva migrans en niños del distrito El Agustino. Rev. Estudiantes de Med. UNMSM 1: 5-10.

17. Servicio Nacional de Metereología e Hidrología (SENAMHI). 2002. Oficina General de Estadística e Informática. SENAMHI. 1 p.

18. Serrano, M.; A. Chávez; E. Casas. 2000. Contaminación de parques públicos del Cono Este con huevos de Toxocara spp. Rev. Inv. Vet. Perú 11: 82-87.

19. Shimizu, T. 1993. Prevalence of Toxocara eggs in sandpits in Tokushima city and its outskirts. J. Vet. Med. Sci. 55: 807- 811.

20. Soulsby, E.J. 1987. Parasitología y enfermedades parasitarias de los animales domésticos. $7^{\mathrm{a}}$ ed. p 150-157. Ed. Nueva Editorial. México.

21. Vásquez, T.S; H.A. Ruiz; B.I. Martínez; M.P. Merlín. 1996. Soil contamination with Toxocara spp. eggs in public parks and home gardens from Mexico City. Bol. Chi. Parasitol. 51: 54-58.

22. Velarde, J.A. 1999. Contaminación de los parques públicos de la Provincia Constitucional del Callao con huevos de Toxocara spp. Tesis de Médico Veterinario. Facultad de Medicina Veterinaria, Univ. Nacional Mayor de San Marcos. Lima. 62 p. 